\title{
Comment on the Berkeley Kinetic Network Model
}

\author{
D. K. DOEKSEN, R. J. J. JONGSCHAAP, and H. KAMPHUIS
}

\author{
Rheology Group \\ Department of Applied Physics \\ Twente University of Technology \\ Enschede \\ The Netherlands
}

\begin{abstract}
A kinetic model for the rheological behavior of polymeric systems, i.e. the Berkeley kinetic network model, is compared with a generalized transient-network model. It turns out that the Berkeley kinetic network model fits quite well in the framework of the transient-network model. From the point of view of the latter model, however, a number of parameters introduced in the kinetic-network model are not independent constants as was supposed. Since the generalized transientnetwork model is totally based on microscopic assumptions, the parameters in this theory reveal information about the structure, whereas the physical interpretation of the parameters in the kinetic model is vague. Stress predictions of the generalized transient-network model agree with experimental data from a 4 percent solution of polystyrene in arochlor, obtained from literature.
\end{abstract}

\section{INTRODUCTION}

C Concentrated solutions and melts of polymers subjected to large deformation rates exhibit nonlinear rheological properties, which in the network theory are assumed to be caused by the existence of a network of entangled polymer molecules. In order to describe the properties of these systems, Soong, Shen, Liu, and Williams developed a kinetic network model (1-5), based on the concept of flow induced variations in entanglement loss and regeneration processes. ${ }^{\circ}$ Under transient-flow conditions the network structure becomes time dependent as a result of unequal rates of the competing kinetic processes. In this model, both a contribution to the stress from the entanglements as well as a contribution from the interaction between the polymer molecules and their surroundings are taken into account. The viscoelastic response of the network is expressed by a contravariant Maxwell constitutive equation the coefficients of which depend on the varying number of entanglements. In simple shear flow the stress due to the interaction indicated as segmental friction, is assumed to be linearly related to the shear rate. This model has proved to be successful in fitting shear stress data from transient shear experiments on solutions of monodisperse polystyrene in arochlor (2). It should be noted that only the kinetic equations are based upon microscopic considerations, while a macroscopic constitutive equation for the stress is proposed independently.

- The model will be referred to as the Berkeley model in this paper.
In the so-called transient-network theories the kinetic equations and the constitutive equations are both based upon microscopic considerations. These theories were first proposed by Lodge and Yamamoto in $1956(6,7)$. Recently, on extending these transient-network theories, a generalized transientnetwork model was introduced $(8,9)$, in which two types of segments are assumed to exist, i.e., fixed segments which form a network as in usual transient-network models, and free segments which are fixed at one end to the network and which behave like chains in bead-spring models. Transitions between the two types of segments may take place. The total number of segments is assumed to be constant. The total stress tensor in a system described by the generalized transient-network model is determined by contributions from the network of fixed segments as well as contributions from the free segments which can be interpreted, as in bead spring models, as a result of the friction between the polymer molecules and the solvent. The function describing the transition process of the segments can be specified in several ways. If we base these functions on the flow-induced concepts of Soong, et al. the results of the generalized transientnetwork model can be compared with the results and the assumptions of the Berkeley model.

\section{THEORY}

\section{The Constitutive Equation}

According to the generalized transient-network model the stress contribution of the polymer mol- 
ecules consists of two parts:

$$
\underline{T}_{p}=\underline{T}_{1}+\underline{T}_{2}
$$

where $T_{1}$ is the stress resulting from the segments that belong to the network and $\underline{T}_{2}$ is the contribution to stress from segments that have been lost from the network.

In this model all segments are considered to behave like Gaussian springs, with modulus $H$ and end-to-end vector $\underline{q}$. So we have:

$$
\underline{T}_{i}=-N_{i} k T \underline{1}+N_{i} H<\underline{q} \underline{q}>_{i} \quad(i=1,2)
$$

$N_{i}$ is the number of segments of type $i$, per unit volume, $k$ is Boltzmann's constant, $T$ the absolute temperature and $\langle.$.$\rangle an average with respect to$ the normalized distribution function of segments of type $i$ :

$$
\int \psi_{i}(\underline{q}, t) d^{3} \underline{q}=1
$$

The balance equations that govern the time dependence of the total segment densities $N_{i} \psi_{i}(\underline{q}, t)$ are equations of continuity in $q$-space, supplemented by terms describing transitions between the two types of segments:

$$
\begin{aligned}
& \frac{\partial N_{1} \psi_{1}}{\partial t}=-\operatorname{div} N_{1} \psi_{1} \underline{\dot{q}}_{1}+g \psi_{2}-h \psi_{1} \\
& \frac{\partial N_{2} \psi_{2}}{\partial t}=-\operatorname{div} N_{2} \psi_{2} \underline{\dot{q}}_{2}-g \psi_{2}+h \psi_{1}
\end{aligned}
$$

The transition parameters $g$ and $h$ are assumed to be independent of $q$. However, they may depend upon global properties such as segmental concentrations, stresses, and the rate-of-strain tensor.

Affine motion is assumed for the fixed segments:

$$
\underline{\dot{q}}_{1}=\underline{L} \cdot \underline{q}_{1}
$$

where $\underline{L}$ is the macroscopic velocity gradient. In the case of free segments, similar to the elastic dumbbell model, we have:

$$
\underline{\dot{q}}_{2}=\underline{L} \cdot \underline{q}_{2}-\left.\frac{2 k T}{\zeta} \frac{\partial}{\partial \underline{q}} \ln \frac{\psi_{2}}{\psi_{0}}\right|_{\underline{q}_{2}}
$$

In this equation $\zeta$ is a friction constant and $\psi_{0}$ the equilibrium distribution:

$$
\psi_{0}=\left(\frac{H}{2 \pi k T}\right)^{3 / 2} \exp \left(-\frac{H q^{2}}{2 k T}\right)
$$

The kinetic equation for the segment concentrations is derived by integrating Eqs 4 and 5:

$$
\frac{d N_{1}}{d t}=\frac{-d N_{2}}{d t}=g-h
$$

By multiplying Eqs 4 and 5 with $q q$, substituting Eqs 6 and 7 and using $E q 2$, constitutive equations for the partial stresses are obtained:

$$
\frac{\delta}{\delta t} \underline{T}_{1}+h_{1} \underline{T}_{1}-g_{2} \underline{T}_{2}=2 N_{1} k T \underline{D}
$$

$$
\frac{\delta}{\delta t} \underline{T}_{2}+\left(g_{2}+\lambda^{-1}\right) \underline{T}_{2}-h_{1} \underline{T}_{1}=2 N_{2} k T \underline{D}
$$

with $g_{i}=g / N_{i}, h_{i}=h / N_{i}(i=1,2)$ and $\lambda=\zeta / 4 H$, a time constant similar to the one in the elastic dumbbell model. The convected derivative is defined as:

$$
\frac{\delta}{\delta t} \underline{A}=\frac{d}{d t} \underline{A}-\underline{L} \cdot \underline{A}-\underline{A} \cdot \underline{L}^{T}
$$

\section{Kinetic Functions}

In order to apply Eqs 9 to 11 , functions $g$ and $h$ still need to be specified. Since we want to discuss the assumptions and predictions of the Berkeley kinetic network model, $g$ and $h$ will be based upon the kinetic assumptions of this model. The creation of fixed segments in this model is assumed to be effective through random thermal diffusion whereas the loss of fixed segments is assumed to be caused by the imposed flow. This results in:

$$
\frac{d N_{1}}{d t}=\frac{K_{c}}{\Lambda^{m}} N_{10}-\frac{K_{c}}{\Lambda^{m}} N_{1}-K_{1} \dot{\gamma}^{m} N_{1}
$$

in which $K_{c}$ and $K_{1}$ are constants, $\Lambda$ is a diffusion time, $m(\leq 1)$ is a parameter sensitive to the elastic nature of the polymer, and $N_{10}$ denotes the equilibrium value ${ }^{* *}$ of the number of fixed segments.

In the Berkeley model the first two terms on the right-hand side of $E q 13$ are considered as a creation function and the last term as an annihilation function. In the present model, however, the creation and loss terms are signed positive and negative respectively and are assumed to have non-zero equilibrium values. Therefore we choose:

$$
\begin{aligned}
& g=\frac{K_{c}}{\Lambda^{m}} N_{\mathrm{l} 0} \\
& h=\left(\frac{K_{c}}{\Lambda^{m}}+K_{\mathrm{l}} \dot{\gamma}^{m}\right) N_{\mathrm{l}}
\end{aligned}
$$

Expressed in equilibrium values and dimensionless parameter sets, Eqs 14 and 15 become:

$$
\begin{aligned}
& g=g_{10} N_{10} \\
& h=h_{10} N_{10}\left(1+\dot{\gamma}^{m}\right) x
\end{aligned}
$$

with:

$$
\begin{aligned}
g_{10} & =h_{10}=\frac{K_{c}}{\Lambda^{m}} \\
\bar{\gamma} & =a \dot{\gamma} h_{10}^{-1} \\
a & =\frac{K_{c}}{\Lambda^{m-1}}\left(\frac{K_{1}}{K_{c}}\right)^{1 / m} \\
x & =\frac{N_{1}}{N_{10}}
\end{aligned}
$$

\section{The Transient Network Limiting Case}

Since the constitutive equation according to the Berkeley model is of the Maxwell type, we consider

$\circ$ The zero index is used in this paper to denote the equilibrium value of the corresponding symbol. 
the limiting case of the generalized transient-network model in which the constitutive equations (Eqs 10 and 11) also reduce to one equation of the Maxwell type. This is the case if the friction coefficient $\zeta$ and thus $\lambda$ are zero; then the free segments adopt their equilibrium distribution instantaneously after creation. It then follows from $E q 7$ that $\psi_{2}=\psi_{0}$, in accordance with the common transientnetwork theories $(6,7)$. Since $\underline{T}_{2}=\underline{0}$, we have $\underline{T}_{p}=\underline{T}_{1}$. In this case $E q 10$ reduces to:

$$
\frac{\delta}{\delta t} \underline{T}_{p}+h_{1} \underline{T}_{p}=2 N_{1} k T \underline{D}
$$

In the Berkeley model the network stress is based upon the Maxwell type constitutive equation:

$$
\frac{\delta}{\delta t} \underline{T}_{p}+\frac{1}{\lambda_{\mathrm{ent}}^{0} \frac{N_{1}}{N_{10}}} T_{p}=2 \frac{\eta_{\mathrm{ent}}^{0}}{\lambda_{\mathrm{ent}}^{0}} \underline{D}
$$

In this equation the parameters $\lambda_{\text {ent }}^{0}$ and $\eta_{\text {ent }}^{0}$ are introduced as constants, which should be fitted independently. Since Eqs 22 and 23 should give an equivalent description of the rheological behavior of the network it follows that $\lambda_{\text {ent }}^{0}$ and $\eta_{\text {ent }}^{0}$ should be related to $h_{1}$ and $N_{1} k T$. From Eqs 14, 15, 22, and 23 we obtain:

$$
\begin{aligned}
& \lambda_{\text {ent }}^{0}=\left(\left(\frac{K_{c}}{\Lambda^{m}}+K_{1} \dot{\gamma}^{m}\right) \frac{N_{1}}{N_{10}}\right)^{-1} \\
& \eta_{\text {ent }}^{0}=N_{1} k T \lambda_{\text {ent }}^{0}
\end{aligned}
$$

We thus see that from the point of view of the transient-network theory the quantities $\lambda_{\text {ent }}^{0}$ and $\eta_{\text {ent }}^{0}$ cannot be considered as independent constants.

\section{MODEL PREDICTIONS}

\section{Linear Viscoelasticity}

The linear viscoelastic behavior is determined by linearization of Eqs 9 to 11 . From Eq 9 it follows that:

$$
g_{20} N_{20}=h_{10} N_{10}
$$

Using Eqs 1 and 26, the complex viscosity is obtained from Eqs 10 and 11 :

$$
\eta^{*}(i \omega)=\frac{\lambda_{1} G_{1}}{1+i \omega \lambda_{1}}+\frac{\lambda_{2} G_{2}}{1+i \omega \lambda_{2}}
$$

with

$$
\begin{aligned}
\lambda_{1,2} & =\frac{1}{2 h_{10}}\left(\alpha \pm \sqrt{\left.\alpha^{2}-4 \lambda h_{10}\right)}\right. \\
G_{1,2} & =\frac{N k T}{2}\left(1 \pm \frac{\alpha+2\left(f_{10}-1\right)}{\sqrt{\alpha^{2}-4 \lambda h_{10}}}\right)
\end{aligned}
$$

In these expressions:

$$
\begin{aligned}
\alpha & =1+\frac{\lambda h_{10}}{1-f_{10}} \\
f_{10} & =\frac{N_{10}}{N}
\end{aligned}
$$

Here $N=N_{1}+N_{2}$ (i.e., a constant) and $f_{10}$ is the equilibrium value of $f_{1}=N_{1} / N$, the fraction of fixed segments. We see that both relaxation times and moduli are related to the model parameters $\lambda, h_{10}$, $f_{10}$, and $N$. If only network chains contribute to the macroscopic stress, i.e., $\lambda=0$, we obtain from $E q s$ 28 to 31 :

$$
\begin{array}{ll}
\lambda_{1}=h_{10}^{-1} & G_{1}=N_{10} k T \\
\lambda_{2}=0 & G_{2}=N_{20} k T
\end{array}
$$

or

$$
\eta^{*}(i \omega)=\frac{h_{10}^{-1} N_{10} k T}{1+i \omega h_{10}^{-1}}
$$

It follows from linearization of the constitutive equation $(E q 23)$ that the linear viscoelastic behavior of the network in the Berkeley model is described by one relaxation time and modulus:

$$
\lambda=\lambda_{\text {ent }}^{0} \quad G=\frac{\eta_{\text {ent }}^{0}}{\lambda_{\text {ent }}^{0}}
$$

where $\lambda_{\text {ent }}^{0}$ and $\eta_{\text {ent }}^{0}$ are now the equilibrium values of the functions 24 and 25 . On substituting these expressions in $E q 35$ we obtain:

$$
\begin{aligned}
\lambda & =\left(\frac{K_{c}}{\Lambda^{m}}\right)^{-1}=h_{10}^{-1} \\
G & =N_{10} k T
\end{aligned}
$$

in accordance with $\mathrm{Eq} 32$.

\section{Viscometric Functions}

In steady shear flow $\left(v_{x}=\dot{\gamma} y, v_{y}=v_{z}=0\right)$ Eqs 9 , 10 , and 11 can be solved explicitly. The following expressions for the viscometric functions are obtained from Eqs 10 and 11:

$$
\begin{gathered}
\eta=\frac{T_{x y}}{\dot{\gamma}}=N k T\left(\left(\frac{f_{1}}{h_{1}}+\frac{\lambda f_{1}}{1-f_{1}}\right)+\lambda\right) \\
\theta=\frac{T_{x x}-T_{y y}}{\dot{\gamma}^{2}}=2 N k T\left(\frac{f_{1}}{h_{1}^{2}}+\frac{\lambda f_{1}}{h_{1}\left(1-f_{1}\right)}\right) \\
+2 \frac{\eta \lambda}{\left(1-f_{1}\right)} \\
\beta=\frac{T_{y y}-T_{z z}}{\dot{\gamma}^{2}}=0
\end{gathered}
$$

The first term on the right-hand side of these expressions is the contribution of the network segments, the last term denotes the contribution of the free segments.

The expressions $f_{1}(\dot{\gamma})$ and $h_{1}(\dot{\gamma})$ are determined from the equation $g_{1}=h_{1}$, which follows from $E q$ 9, and from the assumptions Eqs 16 and 17 :

$$
\begin{aligned}
& f_{1}=f_{10}\left(1+\dot{\gamma}^{m}\right)^{-1} \\
& h_{1}=h_{10}\left(1+\dot{\gamma}^{m}\right)
\end{aligned}
$$

In the transient-network limiting case, i.e., $\lambda=0$, Eqs 38 and 39 with 41 and 42 reduce to:

$$
\eta=\frac{N_{10} k T}{h_{10}\left(1+\bar{\gamma}^{m}\right)^{2}}
$$




$$
\theta=\frac{2 N_{10} k T}{h_{10}^{2}\left(1+\dot{\gamma}^{m}\right)^{3}}
$$

The shear-rate dependence of the viscosity and the first normal stress difference coefficient of the network stress, postulated in the Berkeley kinetic network model, read:

$$
\begin{aligned}
& \eta_{\mathrm{ent}}=\frac{\eta_{\mathrm{ent}}^{0}}{\left(1+\dot{\gamma}^{m}\right)} \\
& \theta_{\mathrm{ent}}=\frac{\theta_{\mathrm{ent}}^{0}}{\left(1+\dot{\gamma}^{m}\right)^{2}}
\end{aligned}
$$

in which $\eta_{\text {ent }}^{0}$ and $\theta_{\text {ent }}^{0}$ are introduced as constants, to be fitted independently. It follows from Eqs 43 and 44 in comparison with Eqs 45 and 46 , that these expressions are not consistent with the proposed form $E q 22$ of the constitutive equation. If we compare the exponents in the denominators of Eqs 43 to 46 it follows again that from the point of view of transient network theory $\eta_{\text {ent }}^{0}$ and $\theta_{\text {ent }}^{0}$ cannot be considered as independent parameters but should be related to the parameters concerning the linear viscoelastic behavior (see also $E q 25$ ):

$$
\begin{aligned}
& \eta_{\text {ent }}^{0}=\frac{N_{10} k T}{h_{10}\left(1+\bar{\gamma}^{m}\right)} \\
& \theta_{\text {ent }}^{0}=\frac{2 N_{10} k T}{h_{10}^{2}\left(1+\dot{\gamma}^{m}\right)}
\end{aligned}
$$

\section{The Contribution of Friction to the Stress}

We now again consider the generalized transientnetwork model in its complete form, i.e., Eqs 9 to 11 , instead of the limiting case $\lambda=0$. In this case the relaxation time $\lambda$ is a parameter concerning the friction between the polymer molecules and the surrounding fluid (see Eqs 7 and 11). So in Eqs 38 and 39 all terms that contain $\lambda$ are contributions of this friction to the viscosity and the first normal stress difference coefficient respectively. In the Berkeley model, a constant term $\eta_{\text {seg }}$ is added to the expression for the shear viscosity $(E q 45)$. This term is related to the frictional stress that chains, according to this model, always carry, whether they are part of the network or dangling. This stress is believed to be purely viscous and proportional to the shear rate because the relevant relaxation time of the chains is always shorter than the time scale for entanglement network dynamics. The microscopic foundation of our expression ( $E q$ 38) is somewhat different. One of the differences between the results obtained in the generalized transient-network model and the assumptions concerning segmental friction in the Berkeley model, is that in the Berkeley model the segmental contributions are constant whereas in Eqs 38 and 39 the terms comprising $\lambda$ are shear-rate dependent. In the Berkeley model the segmental viscosity is attributed to the friction between the polymer chains and the fluid as well as the friction in the entanglements whereas in the generalized transient-network model we only have friction between the free segments and the fluid.

\section{NUMERICAL RESULTS}

This section concerns a comparison between the model predictions of the generalized transient-network model when supplied with the kinetic functions (Eqs 14 and 15) and data reported in Ref. 10 for a solution of 4 percent polystyrene in arochlor. The shear-stress data of the same reference were used by Soong, et al. to evaluate their network model. The values of most parameters in the generalized transient-network model that fit the shearstress data are determined from steady-state viscosity measurements in the same way as was done in Ref. 2. Since the shape of the viscosity curve depends on the parameter $m$ and the dimensionless shear rate $a \dot{\gamma} h_{10}^{-1}$ (see Eqs 38,41, and 42 ), $m$ is estimated from the slope of the viscosity curve in the power law region and $a h_{10}^{-1}$ is estimated from the onset of non-Newtonian behavior. Two other equations concerning parameters can be deduced from the asymptotic solutions of $E q 38$ :

$$
\begin{aligned}
& \lim _{\gamma \rightarrow 0} \eta=N k T\left(\frac{f_{10}}{h_{10}}+\frac{\lambda}{1-f_{10}}\right) \\
& \lim _{\gamma \rightarrow \infty} \eta=N k T \lambda
\end{aligned}
$$

The determination of $N k T \lambda$ from $E q 50$ is not very accurate since the measured values of the viscosity are not constant even at the highest shear rates. Parameters $h_{10}$ and $f_{10}$ are determined from shearstress growth experiments by trial and error, under the condition of $E q 49$. In this process the value of $h_{10}^{-1}$ (dimension time) is found by means of a quantitative fit of the times at which the maxima of the shear stresses occur. The resulting parameter values are listed in Table 1.

The time dependence of the stresses in stress growth experiments, according to the generalized transient-network model, is determined by numerical integration of Eqs 9, 10, and 11 .

Figure 1 shows the steady-shear viscosity as a function of the shear rate and Fig. 2 the shear-stress growth after inception of a steady-shear flow. Both figures also summarize the fits of the Berkeley network model and the experimental data.

Parameters $\lambda, h_{10}^{-1}, f_{10}$, and $N$ of the generalized transient-network model reveal information about the network structure. The value of $h_{10}^{-1}$, i.e., $1.3 \mathrm{~s}$, can be considered as a characteristic time connected with the process of annihilation of network chains. It gives the mean life-time of the network chains at rest. The fraction of all segments that are part of the network and the total number of segments per unit volume are given by $f_{10}$ and $N$ respectively. At rest the system may be considered as a real network for about 70 percent whereas 30 percent consists of free segments.

The relaxation times and storage moduli can be calculated from parameters $\lambda, h_{10}, f_{10}$, and $N$ by using Eqs 28 to 31 . The resulting values are listed in Table 1. They give a global fit of the experimentally determined linear viscoelastic moduli.

Since the generalized network model includes predictions for normal stress differences, the first 


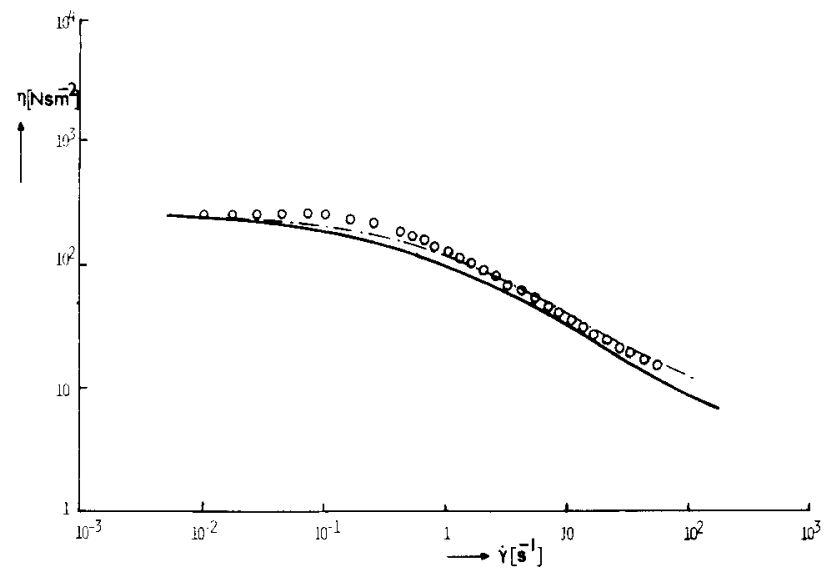

Fig. 1. The shear viscosity as a function of the shear rate for a solution of 4 percent polystyrene in Arochlor at $298 \mathrm{~K}$. (0 measured, - - - calculated from the Berkeley network model using formulas and parameters from (2), - - calculated from the generalized transient-network model using the parameters of Table 1.)

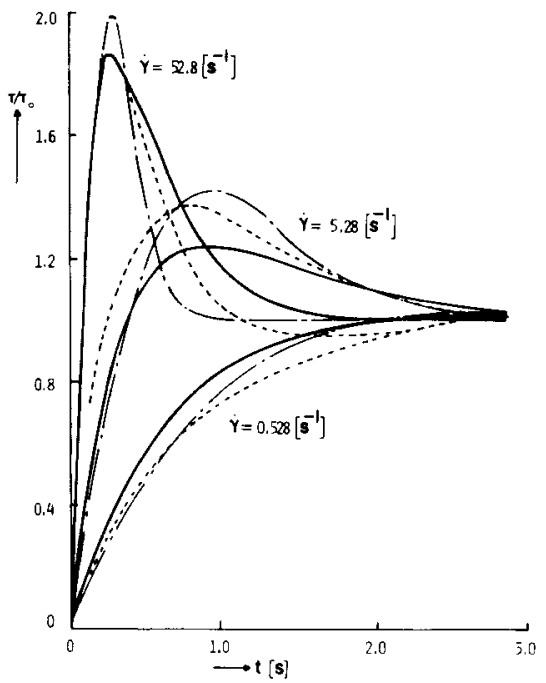

Fig. 2. The dimensionless shear stress of a solution of 4 percent polystyrene in Arochlor after inception of steady-shear flow, at $298 \mathrm{~K}$. (- - measured, - - calculated from the Berkeley network model using formulas and parameters as stated in (2), -_ calculated from the generalized transient-network model using the parameters of Table 1.)

normal stress difference coefficient as a function of the shear rate and the first normal stress difference as a function of time in stress growth and relaxation experiments were also compared with data reported in Ref. 10. These calculations resulted in qualitative fits of the experimental data in which only the shape of the curves is correct.

\section{CONCLUSION}

Using the kinetic assumptions of the Berkeley network model $(1-5)$ in the generalized transientnetwork model $(8,9)$ a set of constitutive equations is obtained which in the transient-network limiting case is similar to the network part of the Berkeley kinetic network model. However, in the generalized transient-network model the parameters in the kinetic expressions, the constitutive equation and
Table 1. Model Parameters from Measurements on a 4 Percent Solution of Polystyrene in Aroclor at $298 \mathrm{~K}$

\begin{tabular}{cl}
\hline Model parameters & $\begin{array}{c}\text { Relaxation times } \\
\text { and moduli }\end{array}$ \\
\hline$\lambda=0.015 \mathrm{~s}$ & $\lambda_{1}=0.015 \mathrm{~s}$ \\
$h_{10}^{-1}=1.3 \mathrm{~s}$ & $\lambda_{2}=1.3 \mathrm{~s}$ \\
$f_{10}=0.7$ & $G_{1}=60 \mathrm{Nm}^{-2}$ \\
$N=5.10^{22} \mathrm{~m}^{-3}$ & $G_{2}=150 \mathrm{Nm}^{-2}$ \\
$a=0.32$ & \\
$m=0.5$ & \\
\hline
\end{tabular}

the expressions derived for the viscosity and the first normal stress difference coefficient are not independent as is assumed by Soong, et al. In using the usual form of the generalized network model we also obtain an expression of the so-called segmental friction. In our case this segmental friction is not a constant as was assumed by Soong, et al but dependent on the shear rate. It has therefore been shown that the basic assumptions in the theory of Soong, et al can be derived from the context of the generalized transient-network model in a consistent way. Moreover since the generalized transient-network model is totally based upon microscopic considerations, the parameters in this model reveal information about the structure of the system whereas the Berkeley model also contains a number of macroscopic parameters. Evaluating the same experimental data as were used by Soong, et al. the generalized transient-network model with the kinetics of the Berkeley model fits the steady-state viscosity and the shear-stress growth experiments. This indicates that these kinetic assumptions give a rather good fit of the actual behavior of the polymeric system. Further progress is expected from the introduction of a spectrum of relaxation times, and from a modification of the assumptions of affine deformation and of the linearity of the force law of segmental springs.

\section{NOMENCLATURE}

$$
\begin{array}{ll}
a & =\text { model parameter }(E q 20) \\
\underline{D} & =\text { rate-of-strain tensor } \\
f_{i} & =\text { fraction of segments of type } i \\
G_{i} & =\text { storage moduli } \\
g, g_{i} & =\text { creation functions } \\
h, h_{i} & =\text { annihilation functions } \\
H & =\text { spring constant } \\
k & =\text { Boltzmann's constant } \\
K_{c}, K_{1} & =\text { model parameters }(E q 13) \\
L & =\text { velocity-gradient tensor } \\
m & =\text { model parameter }(E q 13) \\
N & =\text { total segment density } \\
N_{i} & =\text { density of segments of type } i \\
\underline{q} & =\text { segment vector } \\
t & =\text { time } \\
T & =\text { absolute temperature } \\
T & =\text { macroscopic-stress tensor } \\
x & =\text { structural factor }
\end{array}
$$

Greek symbols

$\alpha \quad=$ dimensionless factor defined in $E q 30$ 
$\dot{\gamma} \quad=$ rate of shear

$\zeta=$ frictional coefficient

$\eta \quad=$ viscosity

$\theta \quad=$ first normal stress difference coefficient

$\lambda, \lambda_{i}=$ relaxation times

$\Lambda \quad=$ model parameter $(E q$ 13)

$\psi \quad=$ segment-distribution function normalized to unity

\section{Subscripts}

$i \quad=$ referring to $i$-th type of segment

$p \quad=$ referring to the polymer molecules

$o \quad=$ referring to equilibrium

Superscript

$-\quad=$ dimensionless variable

\section{REFERENCES}

1. D. S. Soong and M. Shen, J. Polym. Sci., Polym. Lett Ed., 17, $595(1979)$.

2. T. Y. Liu, D. S. Soong, and M. C. Williams, Polym. Eng. Sci, 21, 675 (1981).

3. T. Y. Liu, D. S. Soong, and M. C. Williams, J. Rheol., 27, 7 (1983).

4. T. Y. Liu, D. S. Soong, and M. C. Williams, Am. Chem. Soc, Div. Polym. Chem. Polym. Prepr., 23, 42 (1982).

5. T. Y. Liu, D. S. Soong, and M. C. Williams, submitted to J. Polym. Sci., Polym. Phys. Ed.

6. A. S. Lodge, Trans. Faraday Soc, 52, 120 (1956).

7. M. Yamamoto, J. Phys. Soc. Japan, 11, 413 (1956).

8. R. J. J. Jongschaap, H. Kamphuis, and D. K. Doeksen, Polym. prepr. Am. Chem. Soc., Div. Polym. Chem., 23, 27 (1982).

9. R. J. J. Jongschaap, H. Kamphuis, and D. K. Doeksen, Rheol. Acta, 22, 539 (1984).

10. I. F. MacDonald, Ph.D. thesis, Univ. Wisconsin, Madison (1968). 